\title{
Analysis of HIV quasispecies and virological outcome of an HIV D+/R+ kidney-liver transplantation
}

\author{
Gabriella Rozera ${ }^{1 *}\left(\mathbb{D}\right.$, Ubaldo Visco-Comandini ${ }^{2}$, Emanuela Giombini ${ }^{1}$, Francesco Santini ${ }^{1}$, \\ Federica Forbici ${ }^{1}$, Giulia Berno ${ }^{1}$, Cesare Gruber ${ }^{1}$, Paolo De Paolis ${ }^{3}$, Roberto Colonnelli ${ }^{3}$, Gianpiero D'Offizi ${ }^{2}$, \\ Giuseppe Maria Ettorre ${ }^{4}$, Paolo Grossi ${ }^{5}$, Maria Rosaria Capobianchi ${ }^{1}$, Giuseppe Ippolito ${ }^{6}$ and Isabella Abbate ${ }^{1}$
}

\begin{abstract}
Introduction: Transplantation among HIV positive patients may be a valuable therapeutic intervention. This study involves an HIV D+/R+ kidney-liver transplantation, where PBMC-associated HIV quasispecies were analyzed in donor and transplant recipients (TR) prior to transplantation and thereafter, together with standard viral monitoring.

Methods: The donor was a 54 year of age HIV infected woman: kidney and liver recipients were two HIV infected men, aged 49 and 61. HIV quasispecies in PBMC was analyzed by ultra-deep sequencing of $\mathrm{V} 3$ env region. During TR follow-up, plasma HIV-1 RNA, HIV-1 DNA in PBMC, analysis of proviral integration sites and drug-resistance genotyping were performed. Other virological and immunological monitoring included CMV and EBV DNA quantification in blood and CD4T cell counts.

Results: Donor and TR were all ART-HIV suppressed at transplantation. Thereafter, TR maintained a nearly suppressed HIV-1 viremia, but HIV-1 RNA blips and the increase of proviral integration sites in PBMC attested some residual HIV replication. A transient peak in HIV-1 DNA occurred in the liver recipient. No major changes of drug-resistance genotype were detected after transplantation. CMV and EBV transient reactivations were observed only in the kidney recipient, but did not require specific treatment. CD4 counts remained stable. No intermixed quasispecies between donor and TR was observed at transplantation or thereafter. Despite signs of viral evolution in TR, HIV genetic heterogeneity did not increase over the course of the months of follow up.
\end{abstract}

Conclusions: No evidence of HIV superinfection was observed in the donor nor in the recipients. The immunosuppressive treatment administrated to TR did not result in clinical relevant viral reactivations.

Keywords: HIV, Solid organ transplantation, Quasispecies, Viral reactivation

\section{Introduction}

Kidney transplantation is a primary therapy for end-stage renal disease, just as orthotopic liver transplant (OLT) is considered to be the best curative treatment for patients

*Correspondence: gabriella.rozera@inmi.it

${ }^{1}$ Virology Unit, National Institute for Infectious Diseases, I.R.C.C.S. L.Spallanzani, Via Portuense, 292, 00149 Rome, Italy

Full list of author information is available at the end of the article with hepatocellular carcinoma (HCC) [1, 2]. HIV-positive individuals have a higher incidence of end-stage renal disease (ESRD) and face nearly a threefold higher mortality on dialysis, compared to their HIV-negative counterparts [3-6]. HCV/HIV or HBV/HIV co-infection are frequent in people who inject drugs (PWID) [7, 8]. HCC is a relevant cause of mortality in co-infected patients $[9,10]$, since HIV-related immunosuppression enhances viral replication in liver cells contributing to 
HCC pathogenesis [11]. Advances in combined antiviral therapy (ART) however, have made HIV infection a manageable chronic disease. People currently living with HIV and on ART have a near normal lifespan, and are suitable candidates to receive organ transplant, similar to the general population [12]. HIV+ donor to HIV+ recipient (HIV D $+/ \mathrm{R}+$ ) kidney transplantation was pioneered in South Africa in 2008 [13]. In Italy, HIV infected people became suitable organ donors for HIV positive recipients from 2018. Despite multicenter pilot studies reported that overall patient and graft survival in HIV+ donor to HIV+ recipients were excellent [13-15], the main concern about HIV/HIV transplantation is the possibility of donor derived HIV superinfection of the recipients. Kidneys and livers are considered a reservoir of HIV infection: compartimentalized HIV replication has been demonstrated in kidneys, with site-specific viral variants in urine segregating from those present in plasma [16, 17], whereas livers may harbor latently infected cells in subjects under effective antiviral treatment [18]. Ultradeep sequencing (UDS) of viral quasispecies is a poweful tool to investigate variant mixture among infected individuals and has been used to trace transmission chains and cluster identification $[19,20]$.

The aim of this study was to analyse donor and recipients HIV quasispecies in a $D+/ R+$ kidney-liver transplantation to highlight the possible donor-derived superinfection and to monitor any viral reactivations as well as their clinical consequences.

\section{Methods}

\section{Study population}

The organ donor was a 54-year-old HIV infected deceased woman who had been under suppressive ART since 1997. There was no evidence of viral failure (plasma HIV-1 RNA always under $200 \mathrm{cp} / \mathrm{ml}$ ). Her treatment consisted in darunavir/cobicistat monotherapy with no other documented chronic active viral infections. The cause of death was a spontaneous brain hemorrhage. At the time of organ risk assessment for donation, HIV-1 RNA was not detected in plasma, CD4 count was 951 cells $/ \mathrm{mm}^{3}$, with negative $\mathrm{HBV} / \mathrm{HCV}$ markers.

The kidney recipient was a 49 year-old haemophilic patient with end stage renal disease on hemodialysis; he was infected with HIV (CDC stage B3), HBV (HBsAb+) and $\mathrm{HCV}$ (undetectable HCV-RNA). At the time of transplant, he had been under successfull ART (raltegravir plus rilpivirine) with $\mathrm{HIV}-1 \mathrm{RNA} \leq 50$ copies/ml for almost 10 years. Previous ART included NRTIs, NNRTIs and protease inhibitors, with occasional HIV-1 RNA viral loads up to 800 copies/ml between 2003 and 2008. HIV drug resistance genotype performed on PBMC DNA at the time of transplant showed the presence of both NRTIs and NNRTIs associated resistance mutations (D67D/N, T69T/N, K70R, K103K/R, K219K/Q). The liver recipient was diagnosed with $\mathrm{HCV}$ related cirrhosis and untreatable hepatocellular carcinoma inside Milan criteria. HCV infection was succesfully treated with DAA in 2016. HBV markers were consistent with efficient immune control $(\mathrm{HBcAb}+, \mathrm{HBsAb})$. A good virologic control was obtained with the last ART (darunavir/cobicistat and raltegravir). After transplant, a new ART (emtricitabine/ tenofovir alafenanide and dolutegravir $50 \mathrm{mg}$ BID) based on the previous GRT results was initiated, to avoid drugdrug interactions with post-transplant anti-rejection therapy. HIV drug resistance genotype performed on PBMC DNA at the time of transplant highlighted the presence of only 3 secondary mutations in viral protease (L63P, V77I and I93L). Both recipients signed a written consent for the use of their clinical data and remaining biological samples for research activities. Authorization for use of clinical data and leftover biological samples for research is not needed by our Institute, according to the Italian Personal Data Protection Code (Legislative Decree No. 196 of 30 June 2003) as amended by Legislative Decree No. 101 of 10 August 2018, article 110-bis.

\section{Virological evaluation}

HIV-1 RNA in plasma was measured by Aptima HIV-1 Quant assay (Hologic Inc. San Diego, CA USA). PBMC associated total HIV-1 DNA was quantified as in [21] with a limit of detection of 2.15 Log copies/million PBMC. HIV-1 pol genotyping was performed on PBMC, as previously described [22, 23]. CMV DNA, and EBV DNA quantifications were performed on whole blood while BKV DNA was monitored in plasma and urine by CMV ELITE MGB, EBV ELITE MGB and BKV ELITE MGB kits, respectively on the ELITe InGenius Instrument (ELITech Group S.p.A, Torino, Italia).

\section{Proviral HIV integration site analysis}

Digestion with restriction enzymes of $10 \mu \mathrm{g}$ of PBMC extracted DNA, ligation to double stranded DNA of a linker and semi-nested PCR using primers complementary to both the linker DNA and the long terminal repeat (LTR) end of the HIV provirus were described in [24]. UDS was performed with the shotgun approach by using the Ion Torrent S5 platform (Thermofisher Scientific, Waltham, MA, USA), following the manufacturer protocols. High-quality reads were mapped on HIV-1 reference sequence using BWA v.0.7.12 [https://doi.org/ 10.1093/bioinformatics/btp324]; reads containing LTR sequence were aligned on an HIV-1 reference genome [NCBI Accession Number KO3455.1] and on the Human Reference Genome [GRCh38], discarding all reads that 
mapped on multiple sites with SAMTOOLS software v.1.3.1 [https://doi.org/10.1093/bioinformatics/btp352].

\section{HIV env region UDS and phylogenetic analysis}

Env region amplification was performed on PBMC DNA by nested PCR: the first and the second PCR were carried out with Platinum quality proofreading polymerase (Invitrogen, by Life Technologies, Monza, Italy). Both PCR were composed of 30 cycles $\left(94{ }^{\circ} \mathrm{C}\right.$ for $2 \mathrm{~min}, 94{ }^{\circ} \mathrm{C}$ for $15 \mathrm{~s}$, annealing at $60^{\circ} \mathrm{C}$ for $30 \mathrm{~s}$, extension at $68^{\circ} \mathrm{C}$ for $1 \mathrm{~min}$ or $30 \mathrm{~s}$ and final elongation at $68^{\circ} \mathrm{C}$ for $5 \mathrm{~min}$ ) with the primers described in [20]. Sequencing was performed with the amplicon approach on Ion S5 sequencer, following the manufacturer protocols. The reads were corrected with an in-house developed pipeline described in [20]. Quasispecies complexity of env region was evaluated by Shannon entropy, normalizing for the number of total variants identified in each sample as described in [25].

\section{Results and discussion}

At the time of transplant (September 2019), the organ donor had no detectable HIV-1 RNA in plasma, while the kidney and liver recipients showed $<30$ and 59 HIV-1 RNA copies/ml in plasma samples, respectively. During the follow-up period (September 2019-May 2021), recipients were monitored for viro-immunological parameters of HIV infection and for the major pathogens able to reactivate in TR.

For HIV infection monitoring, together with HIV-1 plasma viremia, PBMC-associated total HIV-1 DNA was evaluated. Quantitative determinations of CMV and EBV viremia in whole blood were performed at regular intervals in both recipients, while BKV DNA measure in plasma and urine was carried out in the kidney transplanted patient.

In Fig. 1, the kinetics of HIV-1 RNA and PBMC-associated HIV-1 DNA in TR, starting from the time of transplant and throughout the whole follow-up period, are shown. In the kidney recipient, although plasma HIV-1 viremia always remained under the clinical threshold of $50 \mathrm{cp} / \mathrm{ml}$, HIV-1 RNA was detected $<30$ copies $/ \mathrm{ml}$ at different time points; in the same period, the HIV cellular reservoir was almost stable (within $<0.5$ Log copies/million cells) [median (IQR) 3.40 (2.92-3.78) copies/million cells]. The liver recipient invariably showed a detectable HIV-1 viremia with values above the clinical threshold of 50 copies $/ \mathrm{ml}$ at different times and experienced transient peaks of HIV-1 DNA with values returning to basal levels at the last time of observation [median (IQR) 3.11 (3.013.73) copies/million cells].

CD4 $\mathrm{T}$ cell counts remained almost stable starting from the time of transplant and all throughout the follow-up period [median (IQR) 312 (240-354) and 272 (257-312) cell $\left./ \mathrm{mm}^{3}\right]$, in kidney and liver TR, respectively.

In order to provide additional evidence of residual HIV replication, proviral HIV integration site analysis was undertaken in PBMC collected at different times in both TR during the follow-up (Table 1). The reads containing the LTR region obtained for each sample, per time point, were median (IQR) 1858 (1113- 2466). In the kidney recipient (panel A) new integration sites were observed in serial samples together with an increase in the frequency of some integration sites, already detected at baseline; other integration sites present at baseline decreased in the frequency or were lost. In the liver recipient (panel B), the integration sites remained the same with almost identical frequencies, throughout the 5 months of follow-up.

In both recipients, drug resistance genotype performed on proviral DNA in PBMC did not change during the follow-up. The kidney recipient experienced a small EBV reactivation with a peak of $3,567 \mathrm{IU} / \mathrm{ml}$ of EBV DNA in blood soon after transplantation and a transient asymptomatic CMV reactivation with a peak value of 12,454 IU/ml CMV DNA in blood after 12 weeks, not requiring specific treatment. BKV DNA in urine and plasma remained undetectable for the entire follow-up period. The liver recipient did not show any CMV/EBV reactivations.

In order to highlight possible transmission of HIV variants from donor to recipients, an extensive phylogenetic analysis by ultra-deep sequencing of HIV env region was performed in donor and recipients at the time of transplant and in TR at different time points during the follow-up period (Fig. 2).

A median (IQR) of 1109 (511-2101) of env corrected sequences was obtained per patient/time point. Regarding viral tropism, all patients carried predominant R5 virus at transplantation and thereafter. The phylogenetic tree constructed with all the corrected sequences from both donor and recipients studied at different times, with respect to the time of transplant, showed complete segregation of HIV quasispecies between the subjects. This implied the absence of donor super-infection of the recipients and an independent genetic evolution in each TR during the follow-up, as suggested by the various sub-clustering observed among each recipient sequences over time. However, this was associated with a decrease of viral complexity overtime, in both TR (see insert in Fig. 2).

Despite the use of a powerful tool able to identify very low minority variants in the HIV quasispecies, this study did not observe any evidence of donor derived variants in $\mathrm{TR}$, as was the case in the recent multicentre study [26]. Moreover, it has to be pointed out that previous studies, which highlighted HIV superinfection of recipients after 
A

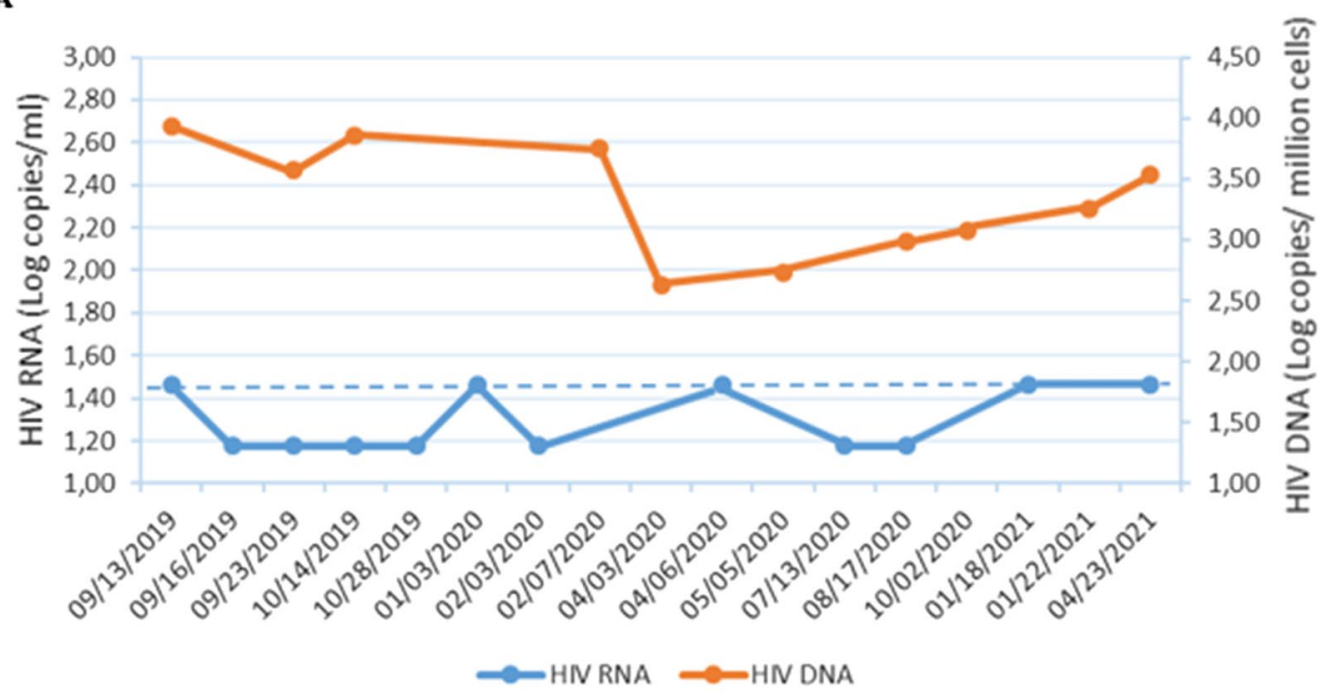

B

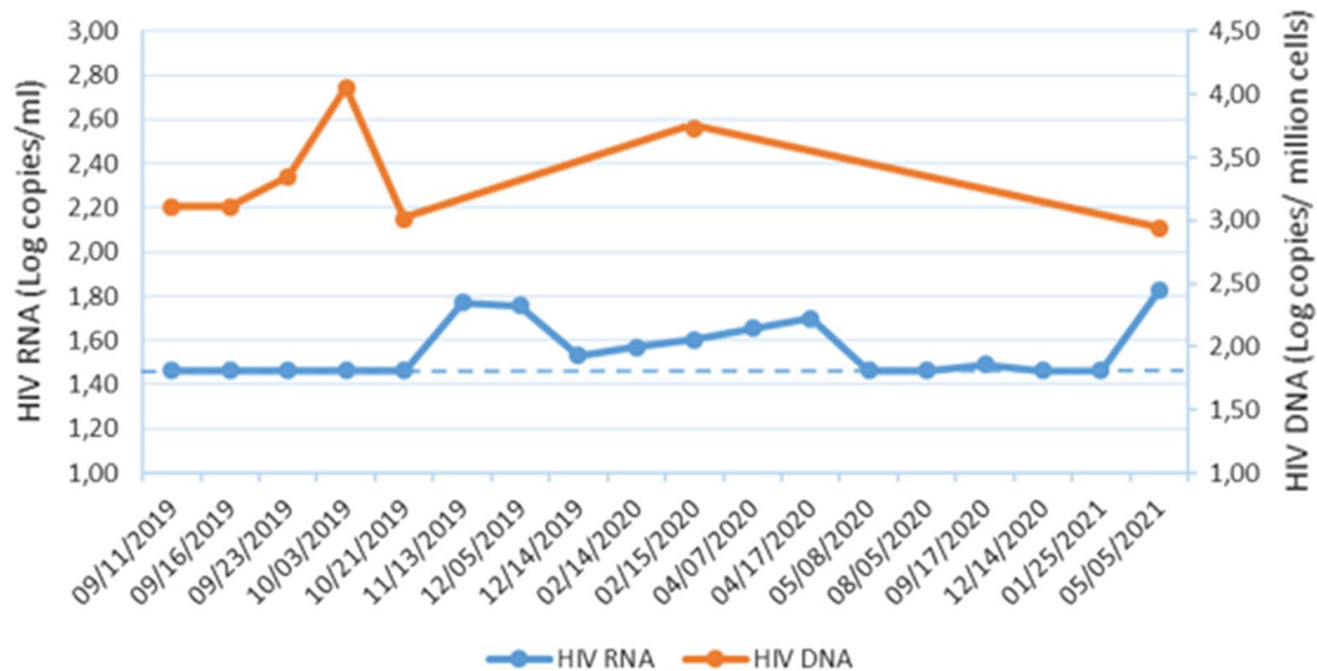

Fig. 1 Kinetics of HIV-1 viremia and peripheral blood cellular reservoir of the infection in TR during the follow-up. Kinetics of HIV-1 RNA in plasma (blue line) and HIV-1 DNA in PBMC (red line) during the follow-up in kidney (A) and liver (B) recipients. The dotted line indicates the limit of quantification of HIV-1 RNA

HIV D+/R+transplantation, mostly involved donors with detectable HIV-1 RNA in plasma [15, 27, 28]. In our case, the organ donor had no detectable HIV-1 RNA in circulation at the time of the organ explant, but it was not possible to rule out a priori the possibility of superinfection of the recipients through the transplanted organs, since both kidney and liver are organ reservoirs of HIV infection. In general, people with HIV superinfection have a less favourable prognosis, displaying lower CD4+ T-cell counts, higher viral loads and a shorter time to adverse clinical events, as compared to mono-infected persons $[29,30]$. In addition, viral recombination in the cells of dually infected persons may occur, resulting in recombinant strains that could be resistant to antiretroviral therapies [31].

In our case, although phylogenetic analysis excluded donor derived superinfection, a low level of HIV replication persisted in both recipients, especially soon after transplantation. This was probably due to the temporary suspension of ART (2-3 days), during the stay in the intensive care unit after transplant. Indirect evidence of residual HIV replication during TR follow-up was the increase in the frequencies or the appearance of new specific types of integration sites, at least in one 
Table 1 Proviral HIV integration sites in the PBMC of the kidney (A) and liver (B) recipients during the post-transplant period

Integration site (chromosome; position)

\section{$\%$ of matches}

09/13/2019

02/07/2020

$01 / 22 / 2021$

A

NC_000015.10; 77401646

NC_000010.11; 21714841

39.74

NC_000005.10; 139967566

29.23

18.24

NC_000001.11; 226567704

3.04

NC_000002.12; 16097405

1.51

NC_000013.11; 59598085

1.28

NC_000011.10; 133588835

1.23

NC_000020.11; 56101682

0.37

NC_000013.11; 28414033

0.26

NC_000014.9; 52551000

0.24

NC_000020.11; 49687838

0.24

NC_000022.11; 46086683

0.15

NC_000004.12; 35007318

0.13

NC_000009.12; 122992409

0.05

NC_000009.12; 74259647

NC_000018.10; 36235516

NC_000004.12; 35007318

NC_000011.10; 58541534

NC_000007.14; 45947801

NC_000016.10; 5408445

NC_000016.10; 72345482

NC_000003.12; 163832295

NC_000001.11; 26998745

NC_000022.11; 46086683

NC_000017.11; 17716854

NC_000001.11; 196452109

NC_000016.10; 5408445

NC_000005.10; 140366572

NC_000001.11; 240464202

0

0

0

0

0

0

0

0

0

0

0

0

0

0

0

Integration site (chromosome; position)

$\%$ of matches

09/23/2019

$\begin{array}{ll}0 & 0 \\ 0 & 0 \\ 54.04 & 57.10 \\ 0.11 & 0.20 \\ 3.34 & 3.61 \\ 0 & 9.37 \\ 0 & 0 \\ 0 & 0 \\ 0 & 0 \\ 0.54 & 0.41 \\ 0.81 & 1.09 \\ 0 & 0 \\ 0 & 0 \\ 0.54 & 0.08\end{array}$

28.09

1.35

0.97

0.81

0.70

0.48

0.43

0

0

0

0

0

0

0

0

0

B

NC_000005.10; 139967566

$\begin{array}{ll}87.96 & 87.64 \\ 4.31 & 4.53 \\ 1.62 & 1.65 \\ 1.26 & 0.82 \\ 1.17 & 1.03\end{array}$

NC_000002.12; 16097405

NC_000020.11; 49687838

NC_000014.9; 52551000

1.17

1.03

The integration sites are reported as human chromosome position; the frequencies for each integration site and for each time point evaluated were calculated as number of reads matched on that site, divided the number of total reads containing a LTR sequence obtained from that sample (\% of matches). Only frequencies deriving from at least 8 matches were considered

patient. Phylogenetic analysis also proved residual HIV replication, since in both TR some viral evolution was observed in the transplant follow-up. Immune-suppressive therapy, administrated to contrast organ transplant rejection, probably played a role in favouring HIV replication. It has been shown that persistent CMV and EBV shedding could contribute to the dynamics of the HIV-1 DNA reservoir during suppressive ART, increasing proviral genetic heterogeneity and HIV disease progression [32, 33]. In this study, CMV and EBV reactivations after transplantation were not associated with an increase of HIV heterogeneity, even in the presence of viral 


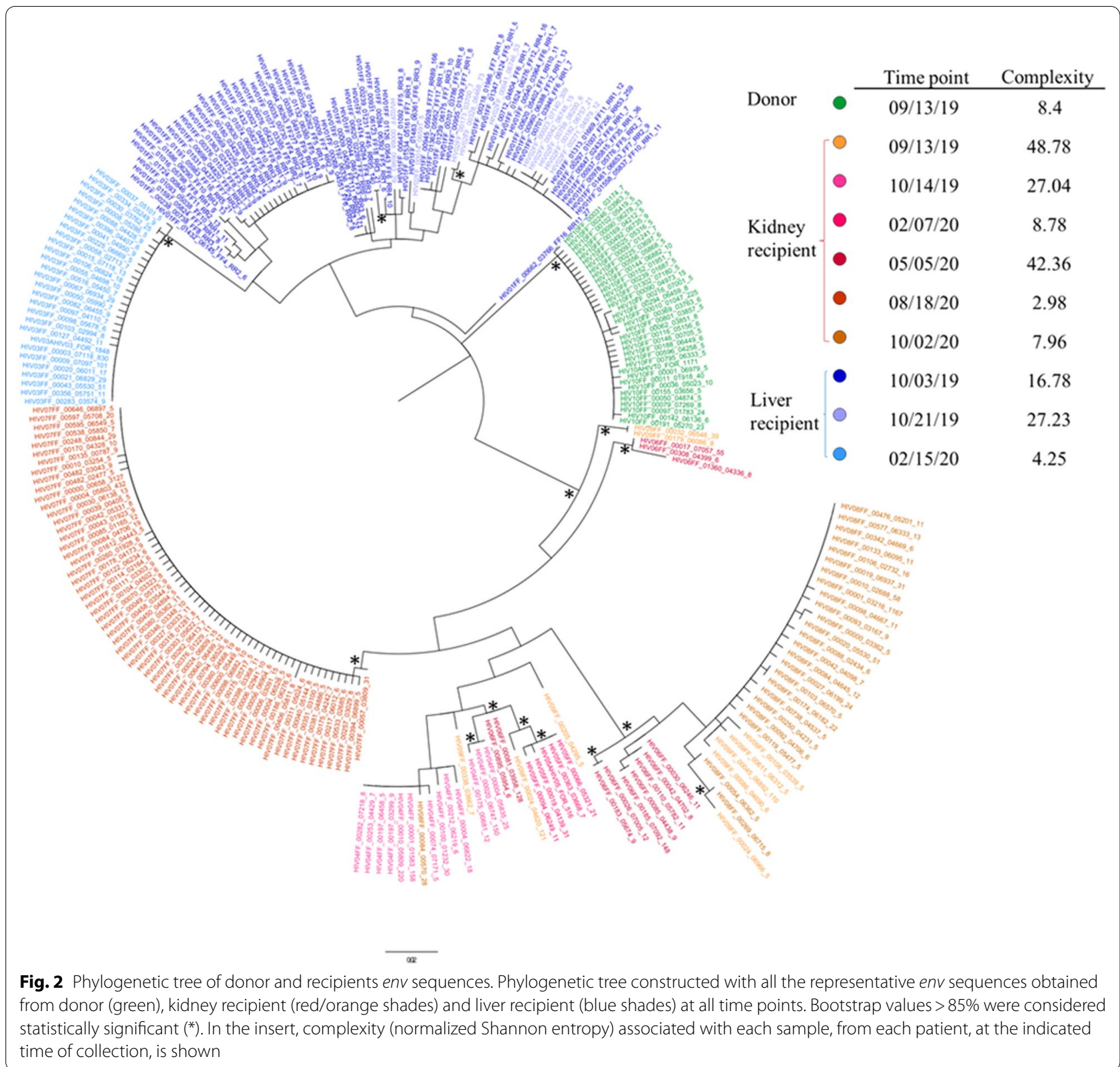

evolution, since in both recipients the HIV-1 DNA quasispecies complexity tended to decrease over time during follow-up.

\section{Conclusions}

No evidence of HIV superinfection was observed in our donor/recipients couple, confirming that HIV D+/ $\mathrm{R}+$ transplantation may be an acceptably safe and valuable therapeutic intervention for HIV infected people. The immunosuppressive treatment provided to minimize transplant rejection, despite being able to promote HIV and other viral reactivations, does not seem to have had a major impact on HIV disease progression. Nevertheless, larger studies on these topics in the future could better consolidate the present conclusions.

\section{Abbreviations}

HIV: Human immunodeficiency virus; TR: Transplant recipients; PBMC: Peripheral blood mononuclear cell; CMV: Cytomegalovirus; EBV: Epstein-Barr virus; BKV: Polyomavirus BK; OLT: Orthotopic liver transplant; HCC: Hepatocellular carcinoma; HCV: Hepatitis C virus; HBV: Hepatitis B virus; ESRD: End-stage renal disease; PWID: People who inject drugs; ART: Antiretroviral therapy; NRTIs: Nucleoside reverse transcriptase inhibitor; NNRTIs: Non nucleoside reverse transcriptase inhibitor; DAA: Direct-acting antivirals; GRT: Genotypic resistance testing; HIV D+/R+ : HIV+ donor to HIV+ recipient; UDS: Ultra-deep sequencing; LTR: Long terminal repeat. 


\section{Acknowledgements \\ Not applicable.}

\section{Authors' contributions}

All authors confirmed they have met the following requirements: (a) significant contributions to the conception and design, acquisition of data, or analysis and interpretation of data; (b) drafting or revising the article for intellectual content; (c) final approval of the published article; and (d) agreement to be accountable for all aspects of the article thus ensuring that questions related to the accuracy or integrity of any part of the article are appropriately investigated and resolved. Contributions of the authors were as follows: I.A. and M.R.C. designed the study; I.A., G.R., and U.V., wrote the manuscript; I.A., G.R., U.V., E.G., F.S., F. F., G.B., C.G., P.D.P., R.C. and G.D. performed the experiments, collected and analysed the data; G.M.E., P.G., and G.I. supervised the whole work. All authors read and approved the final manuscript.

\section{Funding}

This work has been funded by the Italian Ministry of Health (Ricerca Corrente to INMI L. Spallanzani, IRCCS).

\section{Availability of data and materials}

The datasets generated and analysed during the current study are available in the SRA database and will be accessible with the following link, https://www. ncbi.nIm.nih.gov/sra/PRJNA756451

\section{Declarations}

\section{Ethics approval and consent to participate}

The study conformed to the ethical guidelines of the 1975 Declaration of Helsinki. Both recipients signed a written consent for the use of their clinical samples for research activities. Authorization for use of clinical data and leftover biological samples for research is not needed by our Institute, according to the Italian Personal Data Protection Code (Legislative Decree No. 196 of 30 June 2003) as amended by Legislative Decree No. 101 of 10 August 2018, article 110-bis.

\section{Consent for publication}

Not applicable.

\section{Competing interests}

The authors declare that the research was conducted in the absence of any commercial or financial relationships that could be construed as a potential conflict of interest.

\section{Author details}

'Virology Unit, National Institute for Infectious Diseases, I.R.C.C.S. L.Spallanzani, Via Portuense, 292, 00149 Rome, Italy. ${ }^{2}$ Hepatology Unit, P.O.I.T. San CamilloSpallanzani, Rome, Italy. ${ }^{3}$ Nefrology Unit, P.O.I.T. San Camillo-Spallanzani, Rome, Italy. ${ }^{4}$ Surgical Unit, P.O.I.T. San Camillo-Spallanzani, Rome, Italy. ${ }^{5}$ Insubria University, Varese, Italy. ${ }^{6}$ Scientific Direction, National Institute for Infectious Diseases, I.R.C.C.S. L.Spallanzani, Rome, Italy.

Received: 3 September 2021 Accepted: 17 December 2021

Published online: 06 January 2022

\section{References}

1. Abecassis M, Bartlett ST, Collins AJ, Davis CL, Delmonico FL, Friedewald Jر, et al. Kidney transplantation as primary therapy for end-stage renal disease: a national kidney foundation/kidney disease outcomes quality initiative (NKF/KDOQI ${ }^{\mathrm{TM}}$ ) conference. Clin J Am Soc Nephrol. 2008;3:471-80.

2. Chiao H, Edward Yang C, Frenette CT. Review on liver transplant for hepatocellular carcinoma. Transl Cancer Res. 2013:2:472-81.

3. Abraham AG, Althoff KN, Jing Y, Estrella MM, Kitahata MM, Wester W, et al. End-stage renal disease among HIV-infected adults in North America. Clin Infect Dis. 2015;60:941-9.

4. Bickel M, Marben W, Betz C, Khaykin P, Stephan C, Gute P, et al. Endstage renal disease and dialysis in HIV-positive patients: observations from a long-term cohort study with a follow-up of 22 years. HIV Med. 2013;14:127-35.

5. Rasch MG, Helleberg M, Feldt-Rasmussen BO, Kronborg G, Larsen CS, Pedersen C, et al. Increased risk of dialysis and end-stage renal disease among HIV patients in Denmark compared with the background population. Nephrol Dial Transplant. 2014;29:1232-8.

6. Razzak Chaudhary S, Workeneh BT, Montez-Rath ME, Zolopa AR, Klotman PE, Winkelmayer WC. Trends in the outcomes of end-stage renal disease secondary to human immunodeficiency virus-associated nephropathy. Nephrol Dial Transplant. 2015;30:1734-40.

7. Rashti R, Alavian SM, Moradi Y, Sharafi H, Bolbanabad AM, Roshani D, et al. Global prevalence of HCV and/or HBV coinfections among people who inject drugs and female sex workers who live with HIV/AIDS: a systematic review and meta-analysis. Arch Virol. 2020;165:1947-58.

8. Bergo CJ, Epstein JR, Hoferka S, Kolak MA, Pho MT. A vulnerability assessment for a future HIV outbreak associated with injection drug use in Illinois, 2017-2018. Front Sociol. 2021;6:652672.

9. Kim N. Chronic hepatitis B and co-infection chronic hepatitis B and HIV coinfection: a continuing challenge in the era of antiretroviral therapy. Curr Hepatol Rep. 2020;19:345-53.

10. Guardigni V, Toschi A, Badia L, del Turco ER, Salsi E, Cristini F, et al. Patients living with HIV and cirrhosis the risk for hepatocellular carcinoma after direct-acting antivirals for HCV. AIDS. 2021. https://doi.org/10.1097/QAD. 0000000000002973.

11. El Dika I, Harding JJ, Abou-Alfa GK. Hepatocellular carcinoma in patients with HIV. Curr Opin HIV AIDS. 2017;12:20-5.

12. Miro JM, Grossi PA, Durand CM. Challenges in solid organ transplantation in people living with HIV. Intensive Care Med. 2019;45:398-400.

13. Muller E, Kahn D, Mendelson M. Renal transplantation between HIVpositive donors and recipients. N Engl J Med. 2010;362:2336-7.

14. Durand CM, Zhang W, Brown DM, Yu S, Desai N, Redd AD, et al. A prospective multicenter pilot study of HIV-positive deceased donor to HIV-positive recipient kidney transplantation: HOPE in action. Am J Transplant. 2021;21:1754-64.

15. Selhorst P, Combrinck CE, Manning K, Botha FCJ, Labuschagne JPL, Anthony C, et al. Longer-term outcomes of HIV-positive-to-HIV-positive renal transplantation. N Engl J Med. 2019;381:1387-9.

16. Stadtler H, Wescott E, Hughes K, Chang J, Gao F, Klotman M, et al. HIV-1 diversity and compartmentalization in urine, semen, and blood. Medicine. 2020;99:e23063.

17. Hughes K, Chang J, Stadtler H, Wyatt C, Klotman M, Blasi M. HIV-1 infection of the kidney: mechanisms and implications. AIDS. 2021:35:359-67.

18. Ganesan M, Poluektova LY, Kharbanda KK, Osna NA. Liver as a target of human immunodeficiency virus infection. World J Gastroenterol. 2018;24:4728-37.

19. Abbate I, Vlassi C, Rozera G, Bruselles A, Bartolini B, Giombini E, et al. Detection of quasispecies variants predicted to use CXCR4 by ultra-deep pyrosequencing during early HIV infection. AIDS. 2011;25:611-7.

20. Fabeni L, Rozera G, Berno G, Giombini E, Gori C, Orchi N, et al. Molecular transmission dynamics of primary HIV infections in Lazio Region, years 2013-2020. Viruses. 2021:13:176.

21. Rozera G, Fabbri G, Lorenzini P, Mastrorosa I, Timelli L, Zaccarelli M, et al. Peripheral blood HIV-1 DNA dynamics in antiretroviral-treated HIV/ HCV co-infected patients receiving directly-acting antivirals. PLOS ONE. 2017;12:e0187095.

22. Fabeni L, Berno G, Svicher V, Ceccherini-Silberstein F, Gori C, Bertoli A, et al. Genotypic tropism testing in HIV-1 proviral DNA can provide useful information at low-level viremia. J Clin Microbiol. 2015;53:2935-41.

23. Santoro MM, Fabeni L, Armenia D, Alteri C, Di Pinto D, Forbici F, et al. Reliability and clinical relevance of the HIV-1 drug resistance test in patients with low viremia levels. Clin Infect Dis. 2014;58:1156-64.

24. Serrao E, Cherepanov P, Engelman AN. Amplification, next-generation sequencing, and genomic DNA mapping of retroviral integration sites. J Vis Exp. 2016:109:e53840.

25. Gregori J, Salicrú M, Domingo E, Sanchez A, Esteban Jl, Rodríguez-Frías F, et al. Inference with viral quasispecies diversity indices: clonal and NGS approaches. Bioinformatics. 2014;30:1104-11.

26. Bonny TS, Kirby C, Martens C, Rose R, Desai N, Seisa M, et al. Outcomes of donor-derived superinfection screening in HIV-positive to HIV-positive kidney and liver transplantation: a multicentre, prospective, observational study. Lancet HIV. 2020;7:e611-9. 
27. Hathorn E, Smit E, Elsharkawy AM, Elsharkawy AM, Bramhall SR, Bufton SA, Allan S, et al. HIV-positive-to-HIV-positive liver transplantation. N Engl J Med. 2016;375:1807-9.

28. Blasi M, Stadtler H, Chang J, Hemmersbach-Miller M, Wyatt C, Klotman $\mathrm{P}$, et al. Detection of donor's HIV strain in HIV-positive kidney-transplant recipient. N Engl J Med. 2020;382:195-7.

29. Gottlieb GS, Nickle DC, Jensen MA, Wong KG, Grobler J, Li F, et al. Dual HIV-1 infection associated with rapid disease progression. Lancet. 2004;363:619-22.

30. Cornelissen M, Pasternak AO, Grijsen ML, Zorgdrager F, Bakker M, Blom P, et al. HIV-1 dual infection is associated with faster CD4R T-cell decline in a cohort of men with primary HIV infection. Clin Infect Dis. 2012;54:539-47.

31. Redd AD, Quinn TC, Tobian AAR. Frequency and implications of HIV superinfection. Lancet Infect Dis. 2013;13:622-8.

32. Freeman ML, Lederman MM, Gianella S. Partners in crime: the role of CMV in immune dysregulation and clinical outcome during HIV infection. Curr HIV/AIDS Rep. 2016;13:10-9.

33. Gianella S, Letendre S. Cytomegalovirus and HIV: a dangerous Pas de Deux. J Infect Dis. 2016;214:S67-74.

\section{Publisher's Note}

Springer Nature remains neutral with regard to jurisdictional claims in published maps and institutional affiliations.

- fast, convenient online submission

- thorough peer review by experienced researchers in your field

- rapid publication on acceptance

- support for research data, including large and complex data types

- gold Open Access which fosters wider collaboration and increased citations

- maximum visibility for your research: over $100 \mathrm{M}$ website views per year

At $\mathrm{BMC}$, research is always in progress.

Learn more biomedcentral.com/submissions 